\title{
Preliminary results of the design and development of the data acquisition and processing system for the LAGO Collaboration
}

\author{
L. H. Arnaldi ${ }^{* 1}$, D. Cazar ${ }^{2}$, M. Audelo ${ }^{3}$ and I. Sidelnik ${ }^{4}$ for the LAGO Collaboration ${ }^{5 \dagger}$ \\ ${ }^{1}$ Centro Atómico Bariloche and Instituto Balseiro, Comisión Nacional de Energía Atómica \\ (CNEA), S. C. de Bariloche-Argentina \\ ${ }^{2}$ Colegio de Ciencias e Ingenierías "El Politécnico", Universidad San Francisco de Quito, Quito \\ - Ecuador \\ ${ }^{3}$ Facultad de Mecánica, Escuela Superior Politécnica de Chimborazo (ESPOCH), Riobamba - \\ Ecuador \\ ${ }^{4}$ Centro Atómico Bariloche and Instituto Balseiro and Consejo Nacional de Investigaciones \\ Científicas y Técnicas, S.C. de Bariloche, Rio Negro - Argentina \\ ${ }^{5}$ The Latin American Giant Observatory (LAGO), http://lagoproject.net/. \\ See full list of members and institutions at http://lagoproject.net/collab.html.
}

\section{E-mail: arnaldi@cab.cnea.gov.ar}

We present the preliminary results obtained in the development of the new data acquisition system (DAQ) that will be used by the LAGO Collaboration. According to the requirements of the water Cherenkov detectors (WCD) used in LAGO, the new system must be capable of recording fast pulses ( $\sim$ ns) from a photomultiplier (PMT), control the high voltage level applied to it, in addition to monitoring the atmospheric conditions in which the data were taken. We show some of the figures of merit indicating the performance of the new system working with a WCD. The DAQ system is based on a commercial board plus a custom made interface board. The implementation includes scalers, sub-scalers, an automatic baseline correction algorithm, pressure \& temperature sensing, geolocalization, an external trigger and the capability to set and monitor the high voltage applied to the PMT. The flexibility in the design of the system allows to adapt it to different particle detector technologies, such as silicon photomultipliers (SiPMs), resistive plate chambers (RPC) and scintillators. Preliminary results prove the validity, reliability and high performance of the system.

36th International Cosmic Ray Conference -ICRC2019-

July 24th - August 1st, 2019

Madison, WI, U.S.A.

\footnotetext{
* Speaker.

${ }^{\dagger}$ for collaboration list see PoS(ICRC2019)1177
} 


\section{Introduction}

The Latin American Giant Observatory (LAGO) is a collaboration that comes from the association of Latin American (LA) astroparticle researchers [1]. It started in 2006, and it was originally designed to survey the high-energy component of Gamma-Ray Bursts (GRBs) [2]. It is a network of ground-based water Cherenkov detectors (WCDs) located at different altitudes, between sea level and mountain Chacaltaya at more than $5200 \mathrm{~m}$ a.s.l., with a large range of geomagnetic rigidity cut-offs (RC). One of the primary objectives of LAGO is to install WCDs along with different sites in Latin America. At the moment, the Collaboration is composed of ten countries, forming a non-centralized and collaborative network of 25 institutions [3]. It is very important to notice that this is a collaboration to search for space phenomena and understand its physics, also working as an integrated network of science. LAGO operates as a connection of for many universities developing educational projects, helping students to deal with the construction of detectors, data acquisition systems, and data analysis, along with its physics interpretation.

It has been recently shown that WCDs can be used to study the Solar Modulation (SM) of Galactic Cosmic Rays (GCR) and other transient phenomena by measuring the variations of the flux of secondary particles at ground level [4]. Measuring the flux modulation of the GCR at different locations on Earth, which span over a wide range of rigidity cut-offs, using the same type of detectors can provide important information about the global structure of the magnetic cloud filling out the space environment surrounding the Earth. Astroparticle studies, in the context of GRBs [5, 6, 7], space weather and background radiation at ground level [8], are the main scientific objectives of the Latin American Giant Observatory.

Some of the key features of the data acquisition (DAQ) system of the LAGO detectors is the low budget needed to build it, the reliability and versatility. Unfortunately, the FPGA board used until now; the Nexys 2 [9] by Digilent is discontinued, making it very difficult and expensive to maintain WCDs already installed and implement new detectors systems as stated in the development plan of the Collaboration [10]. Given this situation, it was necessary to decide on a plan for replacing the old electronics, trying to take advantage of the state of the art in DAQ systems now available. In this context, the new DAQ concept of all-in-one arose, and we decided on the commercial board Redpitaya STEMLab [11], as it gives us the required flexibility and low cost.

This work describes the new data acquisition system used for measuring the physical quantities that are necessary to perform the analysis of low energy astroparticles at ground level. In section 2 we show the specification of the Redpitaya STEMLab hardware, that makes it suitable for our needs. Section 3 summarizes the architecture system and in 4 there is an overview of the daughterboard employed mostly for slow control. Section 5 shows results on measurements performed with the developed system, and in section 6 we give some final remarks.

\section{The Redpitaya STEMLab board}

The Redpitaya STEMLab board (originally RedPitaya board) is a low-cost ( $\sim 400$ USD) ana$\log$ signal generation/measurement electronics [11]. Figure 1 and Table 1 shows a schematic overview of the STEMLab Redpitaya board and some of the main specifications for the board, respectively. This commercial board is equipped with a fast dual-ADC and a fast dual-DAC, i.e., 


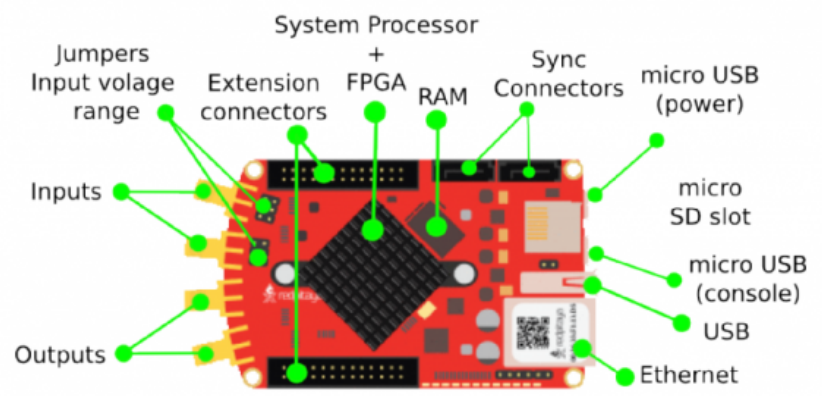

Figure 1: Redpitaya STEMLab board hardware overview [11].

\begin{tabular}{ll}
\hline Processor & $\begin{array}{l}\text { Xilinx Zynq-7010 SoC ARM dual core } \\
\text { CPU and Artix 7 FPGA }\end{array}$ \\
\hline \hline System disk & MicroSD \\
RAM & 512MB \\
Access & USB console, Ethernet, WiFi dongle \\
Power & 5V x 2A max., 0.9A typ. \\
Fast DAC & Dual channel, 14-bit, 125MSPS \\
Fast ADC & Dual channel, 14-bit, 125MSPS \\
Other I/Os & $\begin{array}{l}\text { Slow DAC x 4, slow ADC x 4, digital I/O } \\
\text { x 16, daisy chain connector }\end{array}$ \\
\hline
\end{tabular}

Table 1: Specifications of the Redpitaya STEMLab board

it is easy to use it as a data acquisition system and to generate output signals with an arbitrary phase difference simultaneously. These are the important specifications for its usage as a complete, versatile data acquisition system in the LAGO project. It can run a Linux distribution and can be controlled/accessed via several ways: browsers via PC or tablet (it can run a web server), usb-serial console, and the SSH network protocol. The user can easily write/modify the source code to control the FPGA and the CPU by using open-source software, i.e., it is possible to implement intellectual property (IP) modules on FPGA by using different programming languages, like VHDL or Verilog, and run $\mathrm{C}$ programs on the ARM CPU. Various open-source applications are also available.

\section{System architecture}

The new DAQ system maintains the main characteristics of the former one [12] but incorporates new features that makes it more powerful and optimized to be cost-effective for LAGO. The principal characteristics of the two systems are summarized in Table 2. The new DAQ has a better vertical and horizontal resolution, more data bins per pulse and it runs a Linux distribution. It also has the possibility to implement a network file system (NFS) protocol for data storage and 


\begin{tabular}{l|l|l}
\hline Parameter & Nexys 2 based system & STEMLab based system \\
\hline \hline Vertical resolution & $2 \mathrm{mV}$ & $122 \mu \mathrm{V}$ \\
Horizontal resolution & $25 \mathrm{~ns}$ & $8 \mathrm{~ns}$ \\
Dynamic range & $+1 \mathrm{~V}$ & $\pm 1 \mathrm{~V}$ \\
HV monitoring & N/A & Yes \\
Rate monitoring & N/A & Yes \\
Scaler for pulse rate & Yes & N/A \\
Number of channels & 3 & 2 \\
Number of data bins & 12 (configurable) & 32 (configurable) \\
Number of data bins before trigger & 2 (configurable) & 8 (configurable) \\
NFS ${ }^{1}$ support & N/A & Yes \\
Embedded Linux & N/A & Yes \\
\hline
\end{tabular}

Table 2: Differences between the former LAGO DAQ system based on a Nexys 2 board, and the new one based on Redpitaya STEMLab board. (1) NFS stands for Network File System

includes the monitoring of variables such as the settled high-voltage, the pulse acquisition rate, the temperature at board level and the temperature at detector level.

There is a working mode where the user can set a scaler factor for the maximum frequency of pulses to be acquired. It may be useful in situations where the user needs to acquire a very low level of the signal, and the rate of pulses is huge, exceeding the maximum frequency of data acquisition in normal mode $(\sim 100 \mathrm{kHz})$. In Figure 2 , a block diagram of the DAQ system firmware (FW) is shown. FW is composed of the analog to digital converter managing block (ADC, one per channel), the baseline correction algorithm, the trigger sub-system, the external sensors managing block and all the connections to the external world. All the IP blocks comply with the AXI4Stream standard [13], which is best suited for this application. It follows the Xilinx paradigm in constructing new designs like interconnected intellectual properties blocks.

The DAQ acquires pulses from two channels simultaneously at a sampling rate of 125 MSPS. Each channel can be connected either to one PMT or to the same PMT (anode and last dynode signals) to enhance dynamic range.

The trigger sub-system decides which pulses are stored, based on an absolute threshold settled by the user (in ADC bins) before the start of the data acquisition. In the basic configuration, a total of 32 temporal bins are stored (i.e. $256 \mathrm{~ns}$ at $125 \mathrm{MHz}$ ) for each individual pulse.

\subsection{The baseline correction algorithm}

Generally speaking, a DC offset in the input signal is undesirable because it means that the peaks of the pulses coming from the detectors are more likely to exceed the maximum input level with the consequence of a loss in information. Also, we have to take into account that any change in the level of the baseline could bias the measurements. The knowledge of the baseline level 


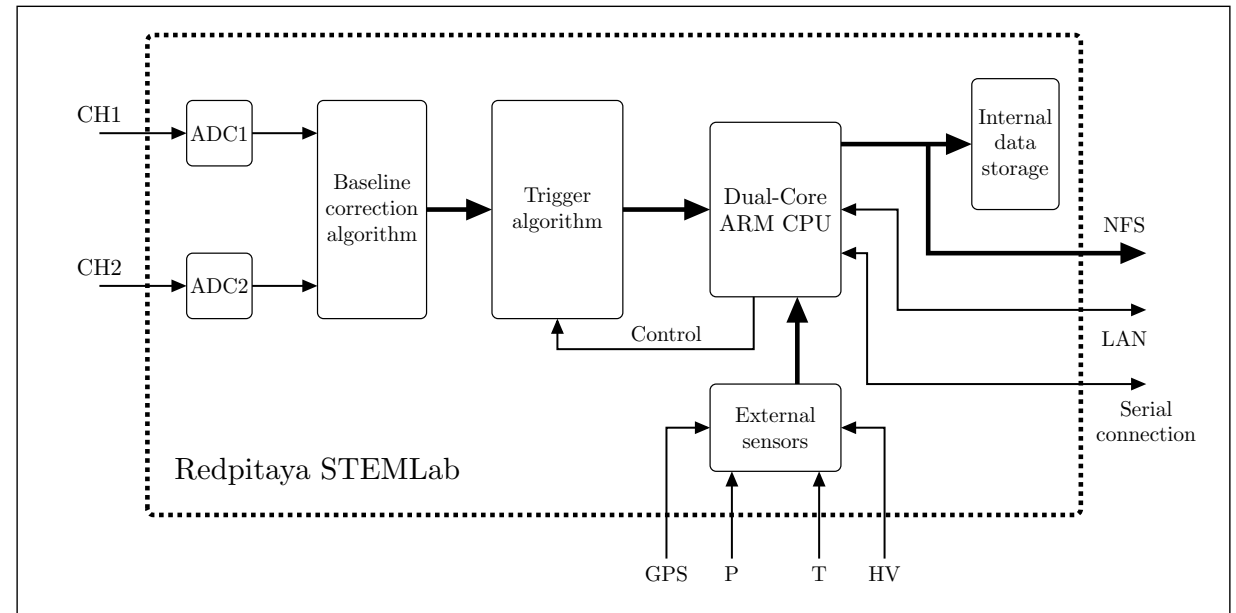

Figure 2: General view of the implemented system. It is shown the different IPs for signal processing and the external sensors for pressure (P), temperature (T), GPS and for high-voltage (HV) monitoring. Also, the two options for data storage, the network filesystem protocol (NFS) and the internal storage block.

is crucial in LAGO, where we intend to measure the rate of particles at ground level with great accuracy.

DC offset should be removed before the analog-to-digital (A/D) conversion of the signal. A dedicated circuit was implemented in the former DAQ system; however, this is not possible here because STEMLab board has a fixed ADC architecture. Therefore, we explore how to remove the DC offset from the digitally sampled waveform using digital signal processing (DSP) without complicated mathematics. We investigate and apply the fractional multiplier technique [14] to create a model of a RC high-pass filter circuit and, therefore, detect the DC level of the input signal digitally and correct it.

A scheme of this implementation is shown in Figure 3, where the fractional multiplier technique is used. We have removed any multiplier in the signal path. Consequently, the baseline correction is done in a very efficient manner (in terms of resource usage and speed). The results of the application of this algorithm in the DAQ's firmware is shown in Figure 4.

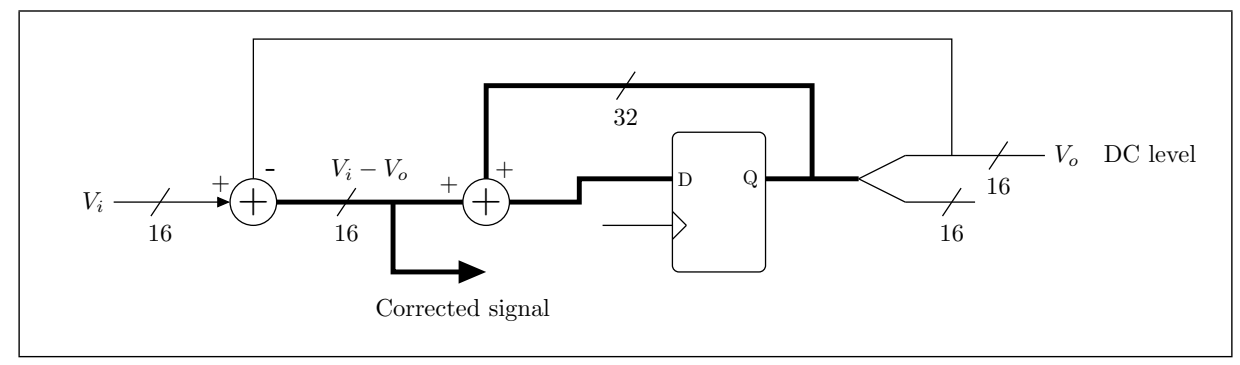

Figure 3: Schematic diagram showing the algorithm for the baseline correction. It is implemented as an intellectual property block in the signal's path.

\section{The daughterboard}

In order to replicate the functionality of the Nexys 2 based DAQ, a "daughterboard" has been 

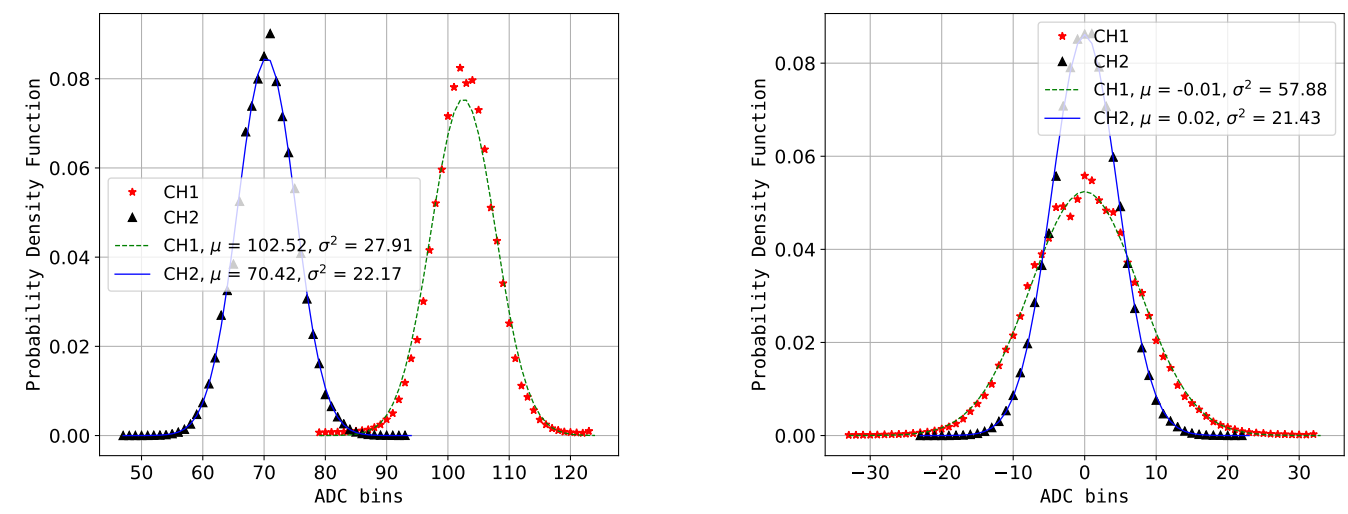

Figure 4: Application of the baseline correction algorithm. The plots show how the baseline correction algorithm works. It centers the baseline at the $0 \mathrm{ADC}$ bin, removing any offset from it and making both channels equal. Baselines without correction (left) and corrected (right).

designed and developed (Figure 5). This board provides different power supply voltages for the internal circuitry of the detectors; it powers the STEMLab board also. It replicates the STEMLab board interfaces to the external sensors and allows the system to be fed through a single external voltage source of $12 \mathrm{~V}$. The board provides the $\pm 3.3 \mathrm{~V}$ for the signal amplifiers in the PMT's base; the control voltage for changing the gain of the photomultiplier tube and the $12 \mathrm{~V}$ for the PMT's high-voltage polarization source. The inter-connection is done through the E1 and E2 connectors in the Redpitaya STEMLab board, where several standard communication protocols (SPI, I2C and UART) are available for plugging a variety of sensors to the system. Schematics, PCB and
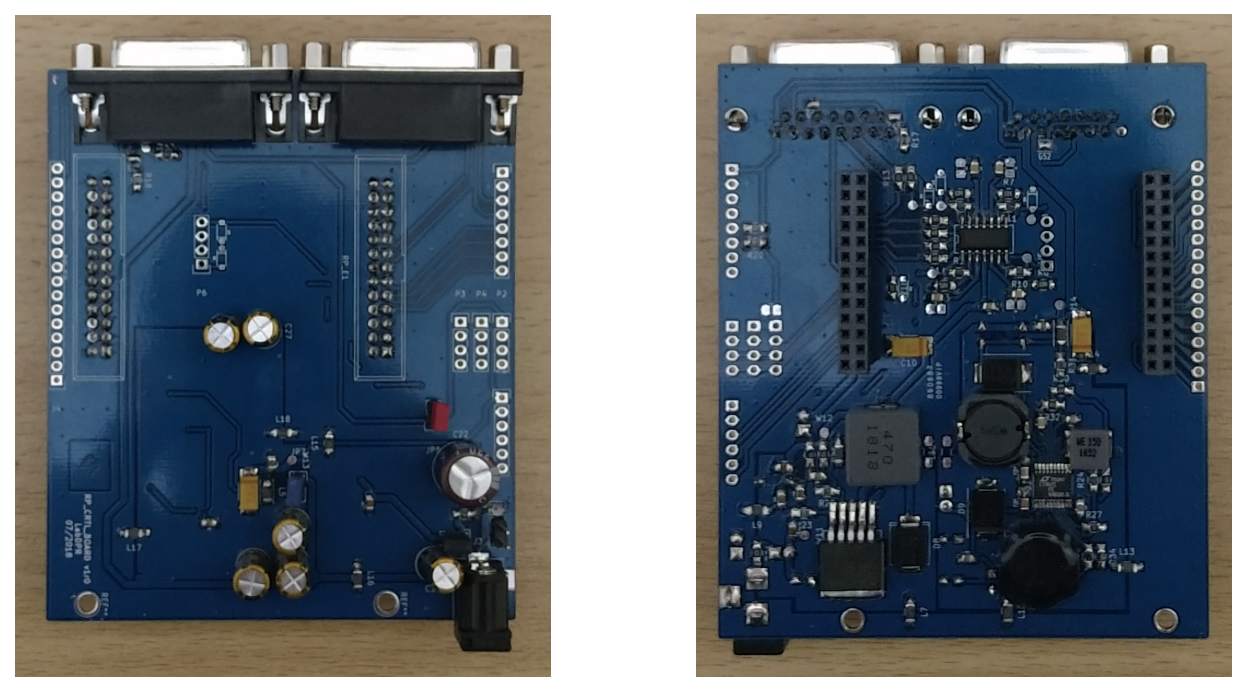

Figure 5: Daughter board for the Redpitaya STEMLab board.

manufacturing files are available in a repository in order to allow any member of the collaboration to build her own system. 


\section{Results of acquisition using the new DAQ}

The system allows us to record different physical quantities that are of interest while measuring with the detectors. We present here some results that show the versatility of the developed system. We show temperature and pressure measurements for atmospheric monitoring and calibration of a WCD with an independent scintillator detector.

\subsection{Coincidence measurements using independent detectors}

One of the clear advantages of this system is that it can control and acquire data from two independent detectors. As a sample of this, we performed a coincidence experiment of a WCD together with a scintillator module. The WCD is a standard LAGO detector with a 9" Photonis XP1805 PMT, and the plastic scintillator module has a 1/2" Hamamatsu R1463 PMT. A diagram of the coincidence scheme can be seen in Figure 6, left. The idea is to test the capability of the developed system for handling both detectors at the same time in slow control as well as in acquisition. The coincidence allows us to calibrate the WCD using traversing muons also detected in the scintillator. An average pulse can be reconstructed from the multiple pulses recorded. This
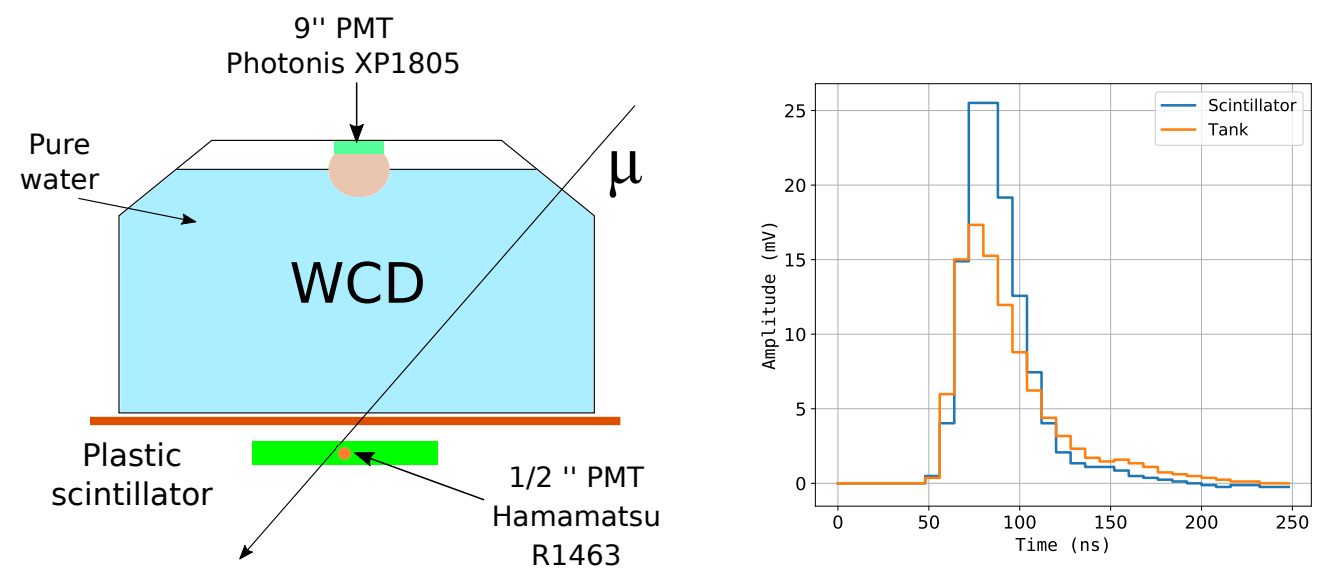

Figure 6: Scheme of the test measurements. There are two detectors, one is a Cherenkov tank with a photomultiplier XP1805, from Photonis and the other is composed of a plastic scintillator working with a photomultiplier R1463, from Hamamatsu (left). The mean pulse from both detectors as seen after an hour of data taking (right).

average pulse gives direct information regarding the propagation of Cherenkov photons within the detector and can be used as a tracer for the water purity and the internal wall reflectivity [12] for the WCD. Figure 6, right, shows the average pulse of the two detectors. It can be seen the difference in intensity but the similar time duration. Building the charge histogram of all recorded pulses allows one to determine the position of the vertical equivalent muon (VEM), the average energy deposited by a through-going central muon, usually appearing as a well-identified peak in the charge histogram. In this situation, we plotted the charge histograms for both detectors. This is depicted in Figure 7.

\section{Conclusions}

In this work, we have shown the upgrade of the data acquisition system used by the LAGO 

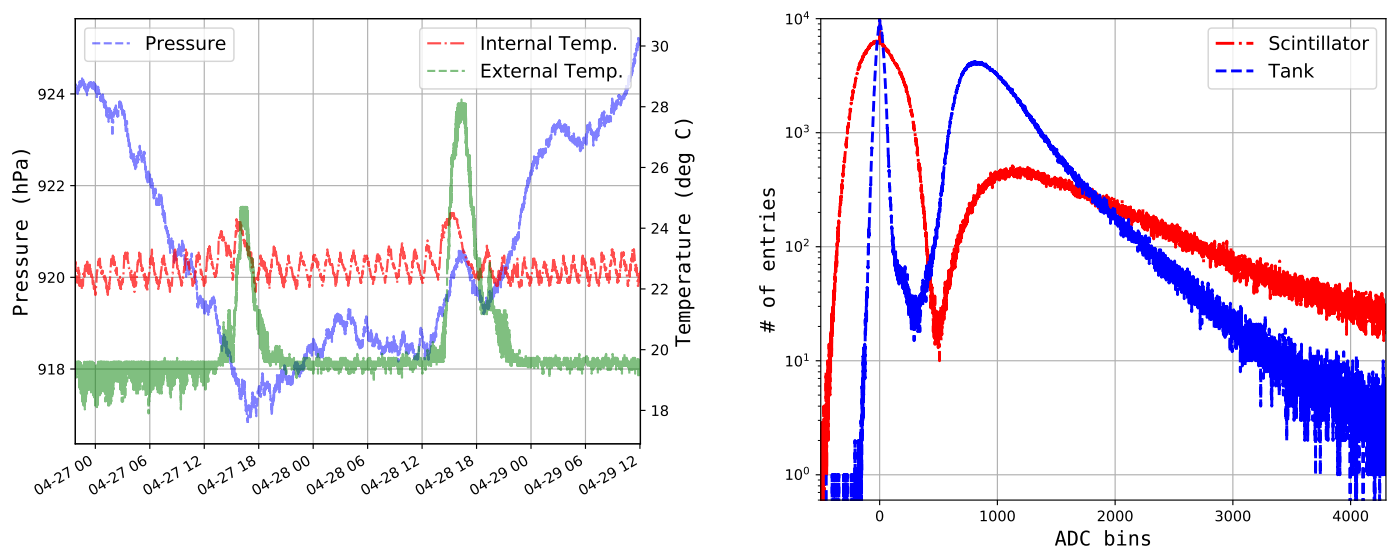

Figure 7: Atmospheric data sensing. The figure shows two days of data taken with the data acquisition system. The different span of the internal and external temperature can be seen (left). Charge histogram of measurements performed with both WCD and scintillator detector (right).

Collaboration. It combines the high resolution ADC found at the Redpitaya STEMLab board and a customized daughter board with the flexibility offered by an FPGA. We have shown how easy it is to adapt it to different particle detector technologies and demonstrated the feasibility and potential of this approach, opening the way for a new generation of data acquisition systems in the field of astroparticle physics. We have shown the main features of the developed system, in addition to some examples of its usage in data analysis.

\section{Acknowledgements}

The authors would like to acknowledge the full support by CONICET and CNEA. This work has been done thanks to the following grants: PICT ANPCyT 2015-2428, PICT ANPCyT 20151644, PICT ANPCyT 2016-2096 and UNCuyo Proy. Cod. 06/C483.

The LAGO Collaboration is very thankful to all the participating institutions and to the Pierre Auger Collaboration for their continuous support.

\section{References}

[1] Asorey H. and Dasso S., for the LAGO Collaboration, LAGO: the Latin American Giant Observatory, in proceedings of the 34th International Cosmic Ray Conference (ICRC 2015), POS ( ICRC2015) 247 (2016).

[2] D. Allard et al., Use of water-Cherenkov detectors to detect Gamma Ray Bursts at the Large Aperture GRB Observatory (LAGO), Nuclear Instruments and Methods in Physics Research Section A: Accelerators, Spectrometers, Detectors and Associated Equipment 595 (Sept., 2008) 70-72.

[3] Latin American Giant Observatory, LAGO: lagoproject . net.

[4] H. Asorey for the LAGO Collaboration, The LAGO Solar Project, in proceedings of the 33th International Cosmic Ray Conference (ICRC 2013),1-4. 
[5] Asorey H., Miranda P., Núñez-Castiñeyra A., Núñez L. A., Salinas J., Sarmiento-Cano C., Ticona R., Velarde A., for the LAGO Collaboration, Analysis of background cosmic ray rate in the 2010-2012 period from the LAGO detectors at Chacaltaya, in proceedings of the 34th International Cosmic Ray Conference (ICRC 2015), POS ( ICRC2015) 414 (2016).

[6] A. Galindo et al. for the LAGO Collaboration, Sensitivity of LAGO and calibration of a water-Cherenkov detector in Sierra Negra, México, in proceedings of the 34th International Cosmic Ray Conference (ICRC 2015).

[7] Otiniano L., Vargas S., Quispe F., Guevara W., for the LAGO Collaboration, Development of a high altitude LAGO site in Peru, in proceedings of the 34th International Cosmic Ray Conference (ICRC 2015).

[8] Asorey H., Dasso S., Núñez L. A., Pérez Y., Sarmiento-Cano C., Suárez-Durán M., for the LAGO Collaboration, The LAGO space weather program: directional geomagnetic effects, background fluence calculations and multi-spectral data analysis, in proceedings of the 34th International Cosmic Ray Conference (ICRC 2015), PoS ( ICRC2015) 142 (2016).

[9] Nexys 2, Nexys 2 Spartan-3E FPGA Trainer Board, Online available: https://store.digilentinc.com/nexys-2-spartan-3e-fpga-trainer-board-retired-see-nexys-4-ddr.

[10] The LAGO Collaboration, The Latin American Giant Observatory: Contributions to the 34th International Cosmic Ray Conference (ICRC 2015), in proceedings of the 34th International Cosmic Ray Conference (ICRC 2015).

[11] RedPitaya STEMLab, RedPitaya Open Source Instrument, Online available: http://www.redpitaya.com/.

[12] M. Sofo Haro and L.H Arnaldi for the LAGO Collaboration, The data acquisition system of the Latin American Giant Observatory (LAGO), Nuclear Instruments and Methods in Physics Research A 820 (2016) 34-39.

[13] ARM, AMBA 4 AXI4-Stream Protocol Specification, Online available: http://www.arm.com/products/system-ip/amba/.

[14] Ken Chapman, WP279 (v1.0) Digitally Removing a DC Offset: DSP Without Mathematics, Xilinx Inc. White Paper, July 2008. 